\title{
Aparición de enfermedades vasculares que alteran la salud de los trabajadores en relación al índice de masa corporal
}

\section{Appearance of vascular diseases that alter the health of workers in relation to the body mass index}

Franklin Hernán Villacis Porras

Universidad Internacional SEK Ecuador, Ecuador

Autor para correspondencia: frankvillacis@hotmail.com

Fecha de recepción: 11 de Julio 2017 - Fecha de aceptación: 30 de noviembre de 2017

\section{Resumen}

El sobrepeso; así como, otras comorbilidades y factores de riesgo se encuentran relacionados con la aparición de enfermedades vasculares y que alteran la salud de los trabajadores que trabajan en posición de pie en jornadas $\geq 8$ horas.

Palabras clave: sobrepeso; enfermedades vasculares; salud de los trabajadores

\begin{abstract}
Overweight; as well as other comorbidities and risk factors are related to the appearance of vascular diseases and that alter the health of workers who work in standing position in days $\geq 8$ hours.
\end{abstract}

Key words: overweight; vascular diseases; workers' health 


\section{Introducción}

A nivel mundial, muchas de las personas en edad económicamente activa, están propensas a generar trastornos de insuficiencia venosa en miembros inferiores (TIVMI), que según su severidad pueden hacerlo en el sistema superficial o en el profundo; así lo recogen estudios precedentes. 1-6

Hoy se reconocen y describen con certeza los factores de riesgo determinantes que se relacionan con la aparición de TIVMI, tales como el sobrepeso, obesidad, el uso de anticonceptivos orales (ACO’s), el estado de gestación, predisposición genética, el desempeño de actividades en bipedestación dinámica o estática por largos períodos de tiempo. 2-4.

La adopción de medidas profilácticas, en personas que trabajan en bipedestación, en jornada laborales igual o mayores a 8 horas y que tengan o no factores de riesgo ayuda a prevenir la aparición signos y síntomas de TIVMI. 3-6

Es por eso que en el presente estudio pretende determinar la relación existente entre la aparición de TIVMI.

\section{Material y métodos}

El presente estudio es de tipo transversal correlacional; se lo llevó a cabo en un grupo heterogéneo de trabajadores entre 18 y 35 años de edad, quienes laboran en bipedestación dinámica, en atención al cliente, en tiendas de artículos deportivos en centros comerciales de la ciudad de Quito - Ecuador, durante jornadas laborales iguales o mayores a 8 horas diarias entre enero 2016 y enero 2017. Se realizó una revisión de fuentes de información secundaria (historias clínicas laborales); para considerarlos como sujetos de estudio, las personas debían de realizan tareas de atención al cliente que involucre la venta de ropa y accesorios deportivos, que como parte de su trabajo mantengan una postura en bipedestación dinámica, que la duración de sus jornadas laborales sean $\geq 8$ horas diarias, que en sus expedientes médicos existan datos de su edad, índice de masa corporal (IMC), antecedentes patológicos familiares (APF), estado de gestación y el consumo de ACO's, que se señalen signos y síntomas de TIVMI como como la presencia de parestesias, edema de predominio vespertino 7,13, telangiectasias, la presencia franca de venas varicosas a nivel de miembros inferiores.

Fueron criterios de exclusión, trabajadores quienes no laboren dentro de la cadena de tiendas en donde se realizó el presente estudio, colaboradores con edades < 18 (en ésta cadena solo laboran mayores de edad) y > 35 años de edad, personal que ingresó a dicha cadena después del 31 de enero 2014, personas con diagnóstico previo de varices. Se ha calculado una $\mathrm{n}=76$ participantes de un universo de 94 personas, con un $\mathrm{IC}=95 \%$ y un porcentaje de error del 5\%; se realizó un análisis correlacional entre las variables de estudio como se muestra en la Tabla 1.

Tabla 1. Correlaciones entre variables de estudio

\begin{tabular}{lrrrrrr}
\hline & & Sexo & Edad & IMC & TIVMI & Gestación \\
\hline Sexo & Correlación de Pearson & 1 & -.165 & .002 & -1.64 & -.081 \\
\hline
\end{tabular}




\begin{tabular}{|c|c|c|c|c|c|c|}
\hline & Sig. (bilateral) & & .155 & .987 & .156 & .488 \\
\hline & $\mathbf{N}$ & 76 & 76 & 76 & 76 & 76 \\
\hline \multirow[t]{3}{*}{ Edad } & Correlación de Pearson & -.165 & 1 &, $381 * *$ & $-.275^{*}$ & -.076 \\
\hline & Sig. (bilateral) & .155 & & .001 & .016 & .511 \\
\hline & $\mathbf{N}$ & 76 & 76 & 76 & 76 & 76 \\
\hline \multirow[t]{3}{*}{ IMC } & Correlación de Pearson & .002 &, $381 * *$ & 1 & -.169 & -.023 \\
\hline & Sig. (bilateral) & .987 & .001 & & .144 & .841 \\
\hline & $\mathbf{N}$ & 76 & 76 & 76 & 76 & 76 \\
\hline \multirow[t]{3}{*}{ TIVMI } & Correlación de Pearson & -.164 & $-.275^{*}$ & -.169 & 1 & .037 \\
\hline & Sig. (bilateral) & .156 & .016 & .144 & & .752 \\
\hline & $\mathbf{N}$ & 76 & 76 & 76 & 76 & 76 \\
\hline \multirow[t]{3}{*}{ Gestación } & Correlación de Pearson & -.081 & -.076 & -.023 & .037 & 1 \\
\hline & Sig. (bilateral) & .488 & .511 & .841 & .752 & \\
\hline & $\mathbf{N}$ & 76 & 76 & 76 & 76 & 76 \\
\hline \multirow[t]{3}{*}{ APF } & Correlación de Pearson & .081 & .076 & .023 & -.037 & .013 \\
\hline & Sig. (bilateral) & .488 & .511 & .841 & .752 & .909 \\
\hline & $\mathbf{N}$ & 76 & 76 & 76 & 76 & 76 \\
\hline \multirow[t]{3}{*}{ ACO's } & Correlación de Pearson &.$^{\mathrm{a}}$ &.$^{\mathrm{a}}$ &.$^{\mathrm{a}}$ &.$^{\mathrm{a}}$ &.$^{\mathrm{a}}$ \\
\hline & Sig. (bilateral) & . & . & . & . & . \\
\hline & $\mathbf{N}$ & 76 & 76 & 76 & 76 & 76 \\
\hline \multicolumn{7}{|l|}{${ }^{*} p<0,05$} \\
\hline$* * \mathbf{p}<0,01$ & & & & & & \\
\hline
\end{tabular}

Los datos obtenidos fueron tabulados con la ayuda del Software SPPS v.24

\section{Resultados}

La distribución de la muestra $(n=76)$ por sexo fue del $67,1 \%$ en hombres $(n=51$ y mujeres el 32,9\% ( $n=25)$ con una edad media de. Los sujetos de estudio, según su edad fueron distribuidos por rangos de edad 65,8\% entre $18-23(n=50), 25,0 \%$ entre $24-29(n=19)$ y 9,2\% entre 30-35 $(n=7)$, como se muestra en la tabla 2.

En relación al peso se halló lo siguiente en relación al IMC, 96,1\% normal (n=73) 7 3,9\% sobrepeso $(n=3)$.

Al realizar un cruce de datos entre sexo y edad, en el rango de edad 18-23 se obtuvo $64,0 \%$ de hombres $(n=32)$ y un $36,0 \%$ de mujeres $(n=18), 24-29$ un $63,2 \%$ de hombres $(n=12)$ y un $36,8 \%$ de mujeres $(n=7)$ y en el de $30-35$ un $100,0 \%$ de hombres $(n=7)$ y un $0,0 \%$ de mujeres $(n=0)$.

Al cruzar datos entre IMC y sexo, con un IMC que corresponde a la normalidad (IMC $=18,5-25)$ un $67,1 \%$ son hombres $(n=49)$ y un $32,9 \%$ son mujeres $(n=24)$; con un IMC que corresponde a sobrepeso (IMC $=25-30)$ un $66,7 \%$ son hombres $(n=51)$ y un $33,3 \%$ son mujeres $(n=25)$. 
Al cruzar las variables TIVMI con sexo, se encontró que un 42,9\% de hombres $(n=3)$ tuvieron algún tipo de signo y/o síntoma de afección vascular en miembros inferiores y un 69,6\% no $(n=51)$; un $57,1 \%$ de mujeres $(n=4)$ tuvieron algún tipo de signo y/o síntoma de afección vascular en miembros inferiores y un $30,4 \%$ no $(n=21)$.

Al cruzar las variables TIVMI con edad, se encontró que entre 18-23 años un 14,3\% si tuvieron algún tipo de signo y/o síntoma de afección vascular en miembros inferiores $(\mathrm{n}=1)$ y un $71,0 \%$ no $(n=51)$, entre $24-29$ años un $71,4 \%$ si tuvieron algún tipo de signo y/o síntoma de afección vascular en miembros inferiores $(n=5)$ y un 14,3\% no $(n=1)$, entre 30-35 años un 14,3\% si tuvieron algún tipo de signo y/o síntoma de afección vascular en miembros inferiores $(\mathrm{n}=1) \mathrm{y}$ un $8,7 \%$ no $(\mathrm{n}=7)$.

Habiendo obtenido los datos más relevantes, se muestra una relación en la Tabla 2.

Tabla 2. Edad*Sexo e IMC*Sexo

\begin{tabular}{llrr}
\hline & & \multicolumn{2}{c}{ Sexo } \\
& & Hombre (n\%) & Mujer $(\mathbf{n \% )}$ \\
\hline EDAD & $\mathbf{1 8 - 2 3}$ & $32(64.0)$ & $18(36.0)$ \\
& $\mathbf{2 4 - 2 9}$ & $12(63.2)$ & $7(36.8)$ \\
& $\mathbf{3 0 - 3 5}$ & $7(100)$ & $0(0.0)$ \\
IMC & Normal & $49(67.1)$ & $24(32.9)$ \\
& Sobrepeso & $2(66.7)$ & $1(33.3)$ \\
\hline
\end{tabular}

Se lleva a cabo una prueba de Chi cuadrado, misma que no se encuentra una relación estadísticamente significativa $(\mathrm{p}<.007)$ como se muestra en la Tabla 3.

Tabla 3. Pruebas de Chi-cuadrado

\section{Valor df Significación asintomática (bilateral)}

\begin{tabular}{lrll}
\hline Chi-cuadrado de Pearson & $9.972 \mathrm{a}$ & 2 & .007 \\
Razón de verosimilitud & 9,276 & 2 & .010 \\
Asociación lineal por lineal & 5,666 & 1 & .017 \\
N de casos válidos & 76 & & \\
\hline
\end{tabular}

\section{Discusión}

Ninguno de los resultados esperados fueron estadísticamente significativos en relación a las variables de estudio y las labores desarrolladas en bipedestación dinámica en jornadas de trabajo $\geq 8$ horas, sin embargo se pudo apreciar que la patología es mayormente predominante en 
hombres que en mujeres entre 18 y 23 años de edad n 32 (64\%) con un peso normal n 49 (67.1\%) aunque otros trabajos se demuestra correlación directa con sobrepeso y obesidad como el realizado por Espínola en 2007 3-6 y el de Caraballo en 2013 efectuado en Venezuela o López Sullaez, Lía Clara. (2015) en su estudio de factores de riesgo ocupacional asociados a las várices en extremidades inferiores.11, 12.

En este estudio no se encuentra asociación estadísticamente significativa entre las variables de estudio, a excepción de la edad y el IMC ( $p>0,01)$, quizá por una tamaño muestras muy pequeño y por falta de recolección adecuada en los datos de las historias clínicas ocupacionales; para estudios posteriores se deberá considerar un periodo de tiempo más largo e incluso otros puestos de trabajo con características similares o que demanden de una posición de pie en jornadas laborales similares; conclusión similar a la que llegan Astudillo et al en su estudio sobre Insuficiencia venosa crónica en trabajadores sin factores de riesgo que permanecen horas prolongadas en bipedestación13.

\section{Bibliografía}

Alfred T. Bazin, M.D., F.A.C.S†. (1929)Varicose VeinsN Engl J Med 1929; 200:442-447February 28,1929DOI: 10.1056/NEJM192902282000910.

Espinóla, Carla Fabiola, Bernal, Manuel, Aucejo, Margarita, \& Villalba, Juan Carlos. (2007). Prevalencia de várices en miembros inferiores en el personal del Hospital de Clínicas. Revista chilena de cirugía, 59(5), 342-347. https://dx.doi.org/10.4067/S071840262007000500006

Joseph N, B A, Faisan Thouseef M, Debi Mu, Abna A, Juneja I. (2016) A multicenter review of epidemiology and management of varicose veins for national guidance. Ann Med Surg (Lond). 2016 Apr 30; 8:21-7. doi: 10.1016/j.amsu.2016.04.024. eCollection 2016. https://www.ncbi.nlm.nih.gov/pubmed/27257482

Robertson, L. a, Evans, C. J., Lee, a J., Allan, P. L., Ruckley, C. V, \& Fowkes, F. G. R. (2014a). Incidence and risk factors for venous reflux in the general population: Edinburgh Vein Study. European Journal of Vascular and Endovascular Surgery: The Official Journal of the European Society for Vascular Surgery, 48(2), 208-14. doi:10.1016/j.ejvs.2014.05.017

Robertson, L. a., Evans, C. J., Lee, a. J., Allan, P. L., Ruckley, C. V., \& Fowkes, F. G. R. (2014b). Incidence and risk factors for venous reflux in the general population: Edinburgh vein study. European Journal of Vascular and Endovascular Surgery, 48, 208-214. doi:10.1016/j.ejvs.2014.05.017

Barrera-Cruz, A., Avila-Jiménez, L., Cano-Pérez, E., Molina-Ayala, M. A., Parrilla-Ortiz, J. I., Ramos-Hernández, R. I., ... Gutiérrez-Aguilar, J. (2013). Practice clinical guideline. Prevention, diagnosis and treatment of overweight and obesity. Revista Médica Del Instituto Mexicano Del Seguro Social, 51(3), 344-57. Retrieved from http://www.ncbi.nlm.nih.gov/pubmed/23883468 
Ng, M., Fleming, T., Robinson, M., Thomson, B., Graetz, N., Margono, C., ... Gakidou, E. (2014). Global, regional, and national prevalence of overweight and obesity in children and adults during 1980-2013: a systematic analysis for the Global Burden of Disease Study 2013. The Lancet, 6736(14), 1-16. doi:10.1016/S0140-6736(14)60460-8

García, J. Fontcuberta, J.J. Samsó, M.E. Senin Fernández R. Coll, Vila Ferrer, y J. M. E. (2014). Actualización de la guía para el diagnóstico no invasivo de la insuficiencia venosa (I). Documento de consenso del capítulo de diagnóstico vascular de la Sociedad Española de Angiología y Cirugía Vascular. Angiología, in press(xx). doi:10.1016/j.angio.2014.05.007

Caraballo-Arias, Yohama, Rodríguez, Arturo Rafael, Rivero, Ángel José, Rangel, Richard Gerardo, \& Barrios Covaro, Marcial. (2013). Riesgos Laborales en Trabajadores de Barberías y Peluquerías de Economía Informal: Caracas, Venezuela. Ciencia \& trabajo, 15(46), 18-23. https://dx.doi.org/10.4067/S0718-24492013000100005

(1997). El edema de miembros inferiores: causas y medidas que lo alivian. Revista Panamericana de Salud Pública, 2(1), 57-58. https://dx.doi.org/10.1590/S1020-49891997000700011

Vázquez, Fernando Javier, Watman, Ricardo, Vilaseca, Alicia B, Rodriguez, Viviana E., Cruciani, Adrián Jorge, Korin, Jorge David, Tabares, Aldo Hugo, Ceresetto, José M., Clavier Lietti, Marcel María, Stinga, Claudia A., Bongiorno, Pablo, Princz, Magdalena Ana, Schutz, Natalia, Barada Palmero, Claudia Eliana, Salvador, Ricardo, Canaveri, Amanda, Morón, Jorge, Pale, Carlos, Saimovici, Javier, Capparelli, Federico J., Wainsztein, Néstor A., Baldessari, Enrique M., Ariscancela, María Ester, \& Lifschitz, Esteban. (2013).

Guía de recomendaciones para la profilaxis de la enfermedad tromboembólica venosa en adultos en la Argentina. Medicina (Buenos Aires), 73(Supl. 2), 1-26. Recuperado en 07 de febrero de 2017, de http://www.scielo.org.ar/scielo.php?script=sci_arttext\&pid=S0025$76802013000700001 \& \operatorname{lng}=$ es\&tlng=es

López Sullaez, Lía Clara. (2015). Factores De Riesgo Ocupacional Asociados A Las Várices En Extremidades Inferiores. Revista Médica La Paz, 21(1), 5-14. Recuperado en 03 de julio de 2017, dehttp://www.scielo.org.bo/scielo.php?script=sci_arttext\&pid=S1726$89582015000100002 \& \operatorname{lng}=$ es\&tlng=es

Astudillo, Paula, Eurgencios, Héctor, Jou, Alicia, \& Solar, David. (2016). Insuficiencia venosa crónica en trabajadores sin factores de riesgo que permanecen horas prolongadas en bipedestación. Medicina y Seguridad del Trabajo, 62(243), 141-156. 